\title{
Voltage Control in Transmission Grids Considering Uncertainties of Renewable Energy Sources_preprint.pdf
}

This paper was downloaded from TechRxiv (https://www.techrxiv.org).

\section{LICENSE}

CC BY-NC-SA 4.0

\section{SUBMISSION DATE / POSTED DATE}

$17-03-2020$ / 17-03-2020

\section{CITATION}

Knittel, Markus; Majumdar, Neelotpal; Schneider, Maximilian; Thie, Nicolas; Moser, Albert (2020): Voltage Control in Transmission Grids Considering Uncertainties of Renewable Energy Sources_preprint.pdf. TechRxiv. Preprint. https://doi.org/10.36227/techrxiv.11994360.v1

$\mathrm{DOI}$ 


\title{
Voltage Control in Transmission Grids Considering Uncertainties of Renewable Energy Sources
}

\author{
Markus Knittel, Neelotpal Majumdar, Maximilian Schneider, Nicolas Thie, Albert Moser \\ Institute for High Voltage Equipment and Grids, Digitalization and Energy Economics \\ RWTH Aachen University \\ Aachen, Germany \\ markus.knittel@rwth-aachen.de
}

\begin{abstract}
Power traders and system operators need to balance the uncertain generation of renewable energy sources by adapting the dispatch of conventional power plants. This poses a challenge to voltage control in power system operation. Accordingly, this paper presents a method to determine voltage magnitude probability densities which are integrated into an optimal reactive power flow to consider an uncertain active power generation. First, the probability densities are determined by Monte Carlo simulations including a unit commitment problem to derive the dispatch of conventional power plants. Second, uncertainty restrictions are used to create soft constraints for the optimal reactive power flow, which mitigates the risk of voltage limit violations. By adapting the soft constraints slack costs, the consideration of uncertainties can be prioritized in relation to other objectives, such as the reduction of active power losses, or reactive power costs.
\end{abstract}

Index Terms - optimal reactive power flow, uncertainty, voltage control

\section{INTRODUCTION}

The progressive integration of renewable energy sources (RES) increases the complexity of voltage control in transmission grids due to weather-based forecast uncertainty of active power generation [1], [2] (Fig. 1).

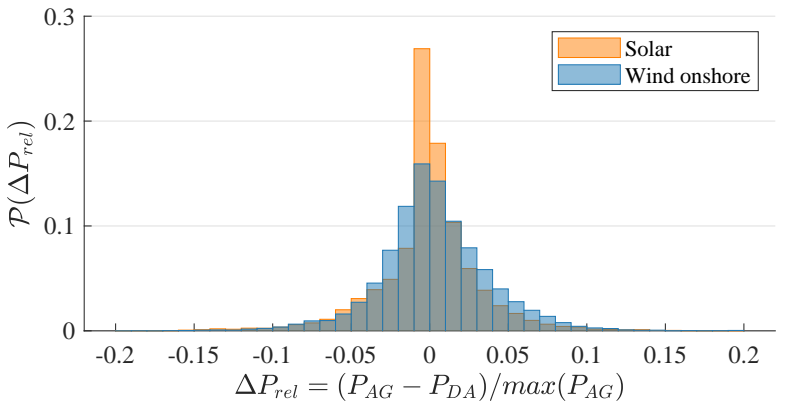

Fig. 1: Day-ahead relative forecast uncertainty for Germany 2018 according to [3], based on the actual generation $P_{A G}$ and day-ahead forecast $P_{D A}$.

To compensate these fluctuations and to maintain the active power equilibrium of power systems, it is necessary to use flexibilities of conventional power plants or loads. In practice, power traders use electric power exchanges, such as the EPEX SPOT for day-ahead or intraday trading, to balance active power consumption and generation. In addition, shortterm imbalances are compensated by different stages of the load-frequency control. These power flow fluctuations also cause an uncertain reactive power demand $Q_{D}$ of lines and transformers, resulting in uncertain voltage magnitudes $V_{M}$ in transmission grids. This aspect has to be considered in operational voltage control by transmission system operators (TSO) to maintain $V_{M}$ within its technical limits. Consequently, a robust dispatch of reactive power compensators must consider these uncertainties in addition to other objectives, for instance the limitation of control actions [4]. Diverse optimal power flow (OPF) formulations have been developed to enable a risk-averse decision-making for RES-driven uncertainties. Many methods, such as the chance-constrained OPF [5], [6] focus on unimodal, symmetrical probability distributions for constraints with zero skewness. Optimal reactive power flow (ORPF) methods have been developed to specifically address voltage control with available reactive power compensators without active power flexibilities [7]. Different adaptions of the ORPF take into account uncertain reactive power demand and compensation [8], [9] and time coupled restrictions [10]. These approaches use pre-defined probability distributions to model uncertainties. However, in order to determine and validate a robust reactive power dispatch, detailed studies on the uncertainty mechanism are required.

This paper presents a method intended for transmission system operation that enables robust reactive power dispatch decisions based on the current system state. It is based on a previously developed ORPF [11], which ignores operational uncertainties. To consider these uncertainties, additional constraints are added to the existing ORPF based on a detailed determination of voltage magnitude uncertainties using Monte Carlo simulations. It is capable of considering uncertainties from RESs leading to non zero skewness or multimodal probability distributions. Voltage uncertainties driven by active power fluctuations are in focus and dependencies between reactive power reserves from conventional power plants and unit commitment are therefore neglected.

\section{MODEL}

The ORPF, which optimizes the dispatch for a number of continuous grid states, is expanded by two steps (Fig. 2). 


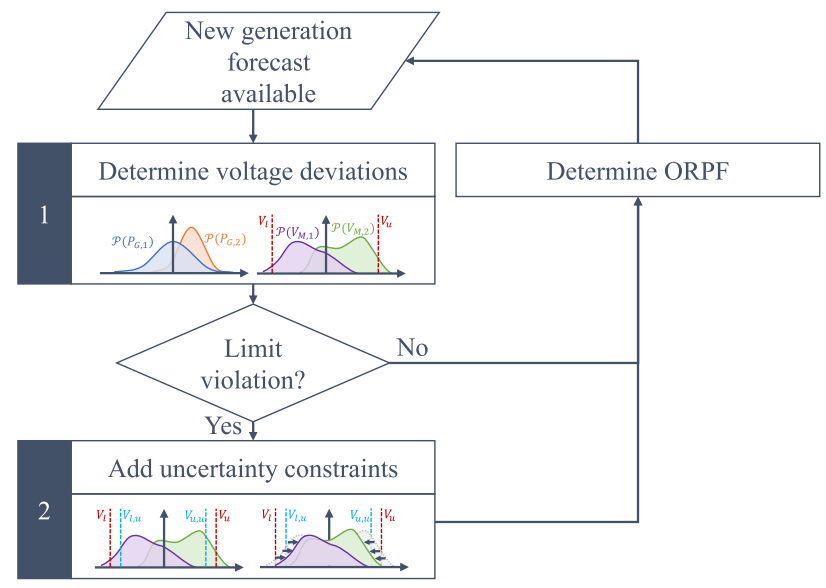

Fig. 2: Model overview.

First, Monte Carlo simulations determine the probability distributions of active power generation $\mathcal{P}\left(P_{G}\right)$ and voltage magnitudes $\mathcal{P}\left(V_{M}\right)$ by using the flexibility of conventional power plants determined by a unit commitment problem (UCP). Second, soft constraints for lower and upper uncertainty bounds $V_{l, u}$ and $V_{u, u}$ are added to the ORPF based on the lower and upper voltage limits $V_{l}$ and $V_{u}$. The violation of these constraints is penalized by a cost term, which is added to the objective function.

\section{A. ORPF (base model)}

Successive Linear Programming (sLP) is chosen as the optimization algorithm for the nonlinear ORPF of the developed method, as it can be solved with robust algorithms like interior point methods. Due to the nonlinear characteristics of grid models, the linearized sensitivities are valid only close to the initial operating point. To avoid this problem, several LP subproblems are solved successively with artificial variable restrictions to achieve a valid solution. After each LP iteration, the results are applied to the grid model and an $\mathrm{AC}$ power flow calculation is carried out to update the operating point and calculate new linear sensitivities. The time-coupled ORPF includes $N_{T}$ grid utilization cases (GUC) between the starting time $t_{b}$ and the end time $t_{e}$. Variables include shunt admittances $\underline{Y}$, reactive power injections $Q$, and ratio tap changers $\tau$ to set voltage controlled devices. The reactive power injected by shunts is voltage dependent and is modeled by means of discrete admittances. Transformer tap changers are modeled with a discrete set of tap-levels. The set of variables $x$ is formulated as (1):

$$
x=\left[\begin{array}{lll}
x^{Q} & x^{Y} & x^{\tau}
\end{array}\right] .
$$

They are classified into open loop and closed loop variables to represent manually switched and automated devices, respectively. While open loop variables are fixed to the same value for all GUCs between $t_{b}$ and $t_{e}$, closed loop values are modeled without any time coupling restrictions. To this end, open loop devices are modeled as a single variable for multiple grid states $x_{b-e}$, whereas closed loop devices are modeled as multiple and independent variables $\left[x_{b}, \ldots, x_{e}\right]$ for each grid state. In total, $N_{x}$ variables are modeled. For all grid states to be optimized, a set of relevant buses is defined comprising $N_{V}$ PQ buses, $N_{Q}$ PV buses and one slack bus. The constraints $b$ yield from the lower and upper voltage magnitude limits at each bus $b_{b-e}^{V l} \in \mathbb{R}^{N_{V} \times 1}$ and $b_{b-e}^{V u} \in \mathbb{R}^{N_{V} \times 1}$ and the lower and upper reactive power limits of each voltage controlled compensator $b_{b-e}^{Q l} \in \mathbb{R}^{\left[N_{Q}+1\right] \times 1}$ and $b_{b-e}^{Q u} \in \mathbb{R}^{\left[N_{Q}+1\right] \times 1}$ (2):

$$
b=\left[\begin{array}{llllll}
-b_{b-e}^{V l} & b_{b-e}^{V u} & -b_{b-e}^{Q l} & b_{b-e}^{Q u} & b^{Q, c} & b^{Q, c}
\end{array}\right]^{T} .
$$

The voltage sensitivities of the variables $A_{b-e}^{V} \in \mathbb{R}^{N_{V} \times N_{x}}$ and the reactive power sensitivities $A_{b-e}^{Q} \in \mathbb{R}^{N_{Q} \times N_{x}}$ are calculated using the system Jacobian. Slack variables $s_{b-e}^{V} \in$ $\mathbb{R}^{1 \times N_{V s}}$ and $s_{b-e}^{Q} \in \mathbb{R}^{1 \times N_{Q s}}$ as well as corresponding costs $c^{V} \in \mathbb{R}^{1 \times N_{V s}}$ and $c^{Q} \in \mathbb{R}^{1 \times N_{Q s}}$ are added to consider initial $N_{V s}$ voltage limit violations and $N_{Q s}$ reactive power limit violations. This means that both voltage and reactive power limits are modeled as soft constraints. While the cost terms for the reactive power injected by shunts $c^{Y}$ and reactive power controlled devices $c^{Q}$ are directly added to the objective function, the cost terms of the voltage controlled devices are added using slack variables $s_{b-e}^{\tau} \in \mathbb{R}^{1 \times N_{Q}}$, the matrix $A^{s, \tau} \in \mathbb{R}^{N_{Q} \times N_{Q}}$ (3), the limits $b^{Q, c}=0$, and the cost term $c^{\tau} \in \mathbb{R}^{1 \times N_{Q}}$

$$
A_{i, j}^{s, \tau}=\left\{\begin{array}{cc}
-1, & \text { if } i=j \\
0, & \text { else }
\end{array}\right.
$$

Furthermore, the matrices $A_{b-e}^{s, V} \in \mathbb{R}^{N_{V} \times N_{V s}}$ and $A_{b-e}^{s, Q} \in$ $\mathbb{R}^{N_{Q} \times N_{Q s}}$ (4) are added to the constraint sensitivities $A(5)$ to consider slack variables:

$$
A_{i}^{s}=\left\{\begin{array}{cc}
-1, & \text { if } b_{i} \text { exceeds limit } \\
0, & \text { else }
\end{array}\right.
$$

$$
A=\left[\begin{array}{ccccccc}
-A_{b-e}^{V} & A_{b-e}^{s, V} & \ldots & \ldots & \ldots & \ldots \\
A_{b-e}^{V} & & \ldots & \ldots & \ldots & \ldots \\
-A_{b-e}^{Q} & \ldots & \ldots & A_{b-e}^{s, Q} & \ldots & \ldots \\
A_{b-e}^{Q} & \ldots & \ldots & & \ldots & \ldots \\
-A_{b-e}^{Q} & \ldots & \ldots & \ldots & \ldots & -A^{s, \tau} & \ldots \\
A_{b-e}^{Q} & \ldots & \ldots & \ldots & \ldots & \ldots & A^{s, \tau}
\end{array}\right]
$$

Variable costs are formed by cost parameters for the injected reactive power and grid losses $c^{L}$. The total cost term for each variable is calculated by (6)-(8):

$$
\begin{gathered}
c^{Q, \text { tot }}=\frac{\Delta P_{\text {Loss }}}{\Delta x_{b-e}^{Q}} \cdot c^{L}+c^{Q}, \\
c^{Y, \text { tot }}=\frac{\Delta P_{\text {Loss }}}{\Delta x_{b-e}^{Y}} \cdot c^{L}+c^{Y}, \\
c^{\tau, \text { tot }}=\frac{\Delta P_{\text {Loss }}}{\Delta x_{b-e}^{\tau}} \cdot c^{L} .
\end{gathered}
$$

Finally, the optimization problem is formulated with cost terms (9), variables (10), the objective function (11), variable limits (12), and constraints (13): 


$$
\begin{aligned}
& c=\left[\begin{array}{llllll}
c^{Q, t o t} & c^{Y, t o t} & c^{\tau, t o t} & c^{V} & c^{Q} & c^{\tau}
\end{array}\right],
\end{aligned}
$$

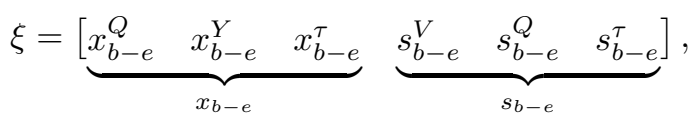

$$
\begin{aligned}
& \min \left(c \cdot \xi^{T}\right) \\
& {\left[\begin{array}{c}
x_{b-e}^{l} \\
0
\end{array}\right] \leq\left[\begin{array}{c}
x_{b-e} \\
s_{b-e}
\end{array}\right] \leq\left[\begin{array}{c}
x_{b-e}^{u} \\
\infty
\end{array}\right]} \\
& A\left[\begin{array}{l}
x_{b-e} \\
s_{b-e}
\end{array}\right] \leq b .
\end{aligned}
$$

\section{B. Monte Carlo simulations}

During system operation, the current system state $t_{0}$ is known while future system states are unknown. A Monte Carlo simulation is used to forecast future system states and to derive robust dispatch decisions [12]. Within these Monte Carlo simulations, all relevant uncertainties (e.g. pv and wind power generation) are sampled to scenarios according to their probability distribution. By using the law of large numbers, the sampled scenarios inherit the same probability distribution as the individual uncertainties. Interdependencies (cross-correlations) between uncertainties, if applicable, are modeled by including the Cholesky decomposition into the sampling. Therefore, the developed method represents the forecast uncertainties for wind and solar based generation by $N_{m c}$ Monte Carlo scenarios (MCS) indexed by $m$. The renewable generation $\sum_{g=1}^{N_{\text {res }}} P_{\text {res }, g, m}$ for each MCS is aggregated with loads $P_{\text {load }}$ and line losses $P_{\text {loss }}$. This represents a residual power demand, which has to be supplied by $N_{c p p}$ conventional power plants. The dispatch of these power plants is derived by a UCP based on [13] using mixed integer linear programming. It models generation and load balancing (14) using equality constraints for $N_{g u c}$ GUCs indexed by $t$ :

$$
\sum_{g=1}^{N_{c p p}} P_{g, t, m}+\sum_{g=1}^{N_{\text {res }}} P_{g, t, m}-P_{\text {load }, t}-P_{\text {Loss }, t}=0 .
$$

The UCP considers fuel-dependent constant and linear cost terms and a distinction between warm and cold start costs. In addition, time coupling is used to model minimal upand downtimes as well as ramping limitations. As a result, a scenario and time series $P_{g, t, m}$ (15) for each active power generation indexed by $g$ is derived for each MCS and GUC.

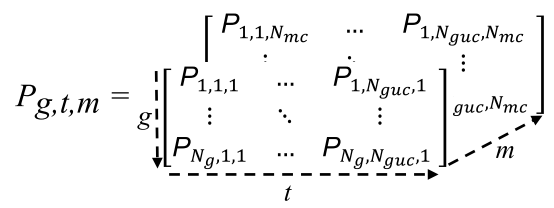

Next, a continuation power flow (CPF) based on [14], [15] determines the voltage magnitude probability distributions for each future grid state. To this end, the current grid state at $t_{0}$ is set as base case. A target case for each MCS is created by setting the corresponding power generation and demand. Fig. 3 visualizes the Monte Carlo simulation for target cases at $t \in\left[t_{1}, \ldots, N_{g u c}\right]$ and MCSs $m \in\left[m_{1}, \ldots, N_{m c}\right]$. The CPF is used instead of conventional power flow algorithms, such as the Newton-Raphson method, to shift the current solution of the base case iteratively. This ensures that the solutions of the target cases are based on the base case solution.

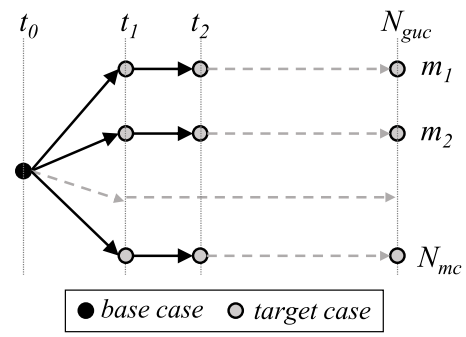

Fig. 3: Continuation power flow target cases.

The CPF maintains the initial settings for voltage references, tap ratios, and the dispatch of reactive power compensators while shifting the active power generation and demand and reactive power demand iteratively from base to target case. Thus, a detailed forecast for voltage magnitudes at all buses is derived. These voltage magnitudes form the probability distribution $\mathcal{P}\left(V_{M, t}\right)$ for each GUC, which is used to integrate uncertainty constraints into the ORPF.

\section{ORPF considering voltage magnitude uncertainties}

The ORPF is extended by adding soft constraints, which consider the maximal and the minimal forecasted voltage magnitude. For each $t \in\left[t_{b}, \ldots, t_{e}\right]$ an upper uncertainty limit $b_{b-e}^{V u, u}(16)$ and a lower uncertainty limit $b_{b-e}^{V l, u}(17)$ are formulated. Alternatively, it is possible to set any confidence interval instead of using the full confidence interval between $\min \left(\mathcal{P}\left(V_{M, b-e}\right)\right)$ and $\max \left(\mathcal{P}\left(V_{M, b-e}\right)\right)$.

$$
\begin{gathered}
b_{b-e}^{V u, u}=2 \cdot b_{b-e}^{V u}-\max \left(\mathcal{P}\left(V_{M, b-e}\right)\right) \\
b_{b-e}^{V l, u}=2 \cdot b_{b-e}^{V l}-\min \left(\mathcal{P}\left(V_{M, b-e}\right)\right)
\end{gathered}
$$

Next, these limits are added to (2) to form the extended constraints (18). The matrix $A_{u}$ (19) is derived by expanding (5) with $A^{s, V, u}=A^{s, V}$. By adding $c^{V, u}$ to (9) the cost terms are expanded to (20) and violations of uncertainty constraints are penalized. The variation of $c^{V, u}$ enables the prioritization of risk-averse voltage control in relation to other costs, such as dispatch costs or active power losses.

$$
\begin{aligned}
b_{u} & =\left[\begin{array}{lll}
b & -b_{b-e}^{V l, u} & b_{b-e}^{V u, u}
\end{array}\right]^{T} \\
A_{u} & =\left[\begin{array}{ccc}
A & \ldots & \ldots \\
-A_{b-e}^{V} & A_{b-e}^{s, V} \\
A_{b-e}^{V}
\end{array}\right]
\end{aligned}
$$




$$
c_{u}=\left[\begin{array}{ll}
c & c^{V, u}
\end{array}\right]
$$

Finally, slack variables $s_{b-e}^{V, u}$ are added to formulate (21), an updated objective function (22), and new variable limits (23):

$$
\begin{aligned}
& \xi_{u}=\left[\begin{array}{lll}
x_{b-e} & s_{b-e} & s_{b-e}^{V, u}
\end{array}\right], \\
& \min \left(c \cdot \xi_{u}^{T}\right), \\
& {\left[\begin{array}{c}
x_{b-e}^{l} \\
0 \\
0
\end{array}\right] \leq\left[\begin{array}{c}
x_{b-e} \\
s_{b-e} \\
s_{b-e}^{V, u}
\end{array}\right] \leq\left[\begin{array}{c}
x_{b-e}^{u} \\
\infty \\
\infty
\end{array}\right]}
\end{aligned}
$$

These are subject to the constraints (24):

$$
A_{u}\left[\begin{array}{c}
x_{b-e} \\
s_{b-e} \\
s_{b-e}^{V, u}
\end{array}\right] \leq b_{u} .
$$

Thus, the adapted formulation considers uncertainties from RESs and enables the determination of a robust reactive power dispatch.

\section{CASE STUdy}

The method is studied for an adapted version of the IEEE 118 bus test system, using a load and generation scenario for 24 hours (Fig. 4) [16]. In total, 17 mechanically switched reactors, 16 mechanically switched capacitors, and 5 STATCOMs are installed as reactive power compensators. HVDCterminals and conventional power plants provide additional reactive power reserves. To model the forecast uncertainty, the generation time series is sampled with $N_{m c}=50$. Cost parameters are chosen as listed in Table I.

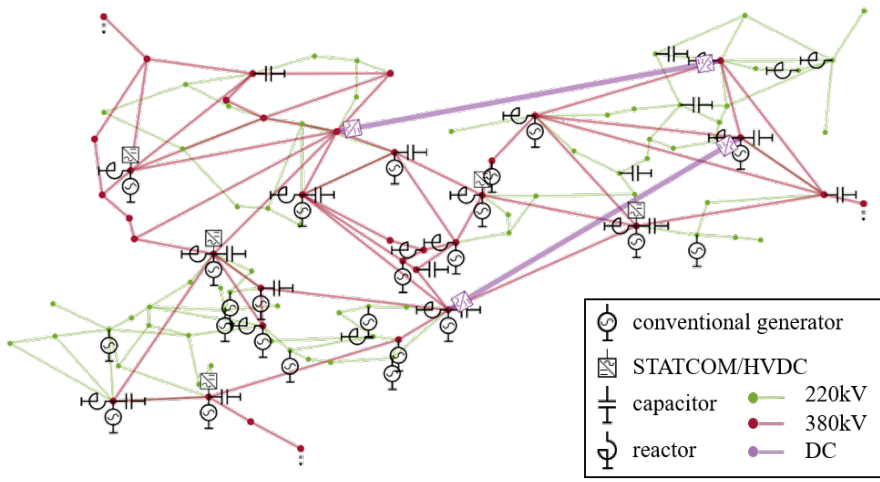

Fig. 4: Test system topology with reactive power compensators

TABLE I: Cost terms

\begin{tabular}{|c|c|c|c|c|}
\hline$c^{L}$ & $c^{Y}$ & $c^{Q}$ & $c^{\tau}$ & $c^{V, u}$ \\
\hline 35 & 0.0025 & $0.05 \mathrm{~s}$ & 0.1 & 500 \\
\hline
\end{tabular}

The reactive power dispatch is determined by the ORPF for continuous GUCs, comprising one known initial grid state and 3-4 future states, modeled by MCSs (Fig. 5). E.g. $t_{1}$ is set as base case and $50 \mathrm{MCSs}$ for $t_{2}-t_{5}$ are set as target cases to be analyzed by the CPF. This models the operational voltage control with the objective to set a robust reactive power dispatch for the current system state and the next 3-4 hours.

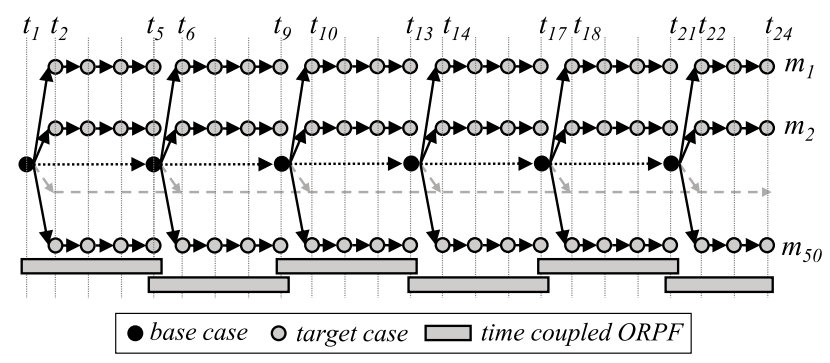

Fig. 5: Base and target cases for the case study.

\section{A. Active Power Generation Uncertainty}

For given probability distributions of renewable power generation uncertainty, the UCP determines the corresponding uncertainty of conventional power plants. The uncertainties can be modeled by a normal distribution (Fig. 6), when aggregated for all conventional and renewable power plants.

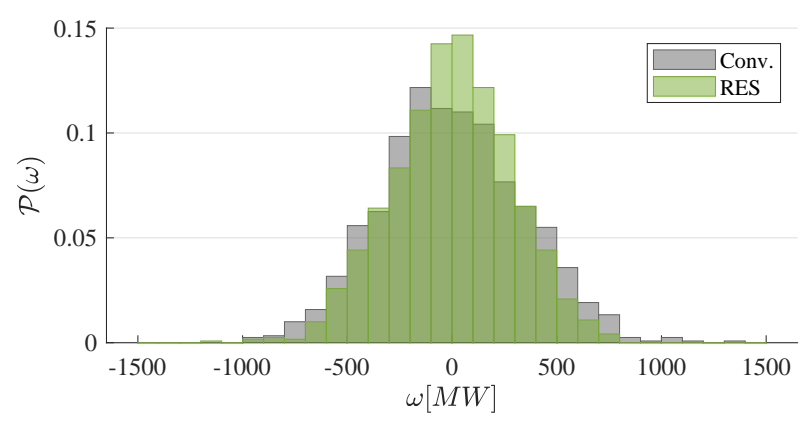

Fig. 6: Forecast probability distributions of renewable and conventional generation $\mathcal{P}(\omega)=\mathcal{P}(P)-\operatorname{mean}(\mathcal{P}(P))$.

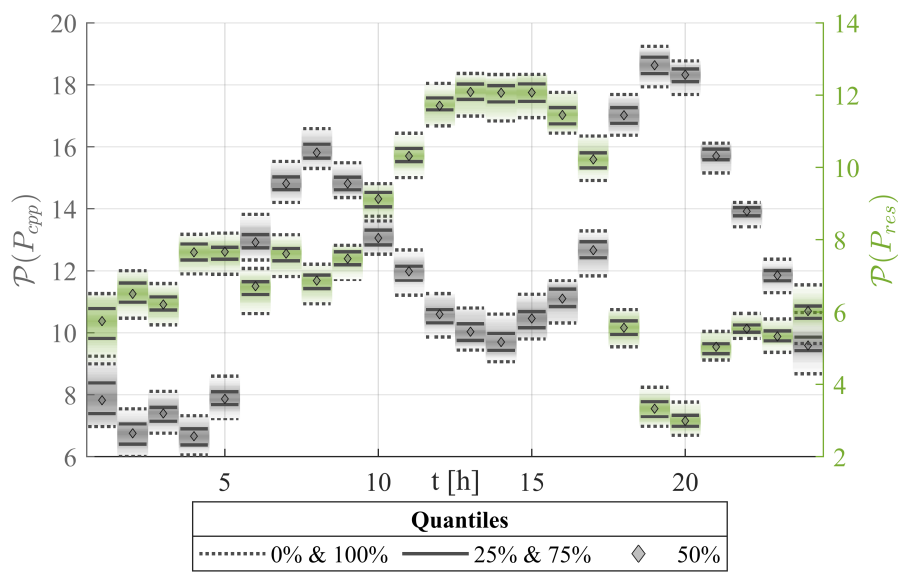

Fig. 7: Conventional and renewable power plant uncertainties.

The fluctuation of power plant types during all GUCs is shown in Fig. 7. Although the system is only reaching a renewable share of $55 \%$, the scale of active power uncertainties from RESs is effectively doubled due to the required dispatch adaption of conventional power plants. A detailed analysis of all MCSs for an exemplary conventional and a wind power plant shows that normal distributions cannot always be used to model the uncertainty of individual generators (Fig. 8). 


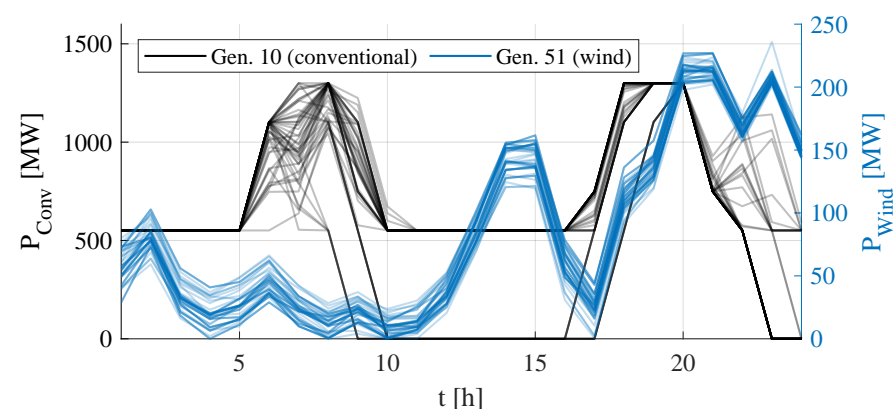

Fig. 8: Exemplary uncertainties.

Renewable active power generation in transmission grids is injected by a high number of individual power plants (e.g. a wind park). It is not constrained by a significant difference between no generation and minimum generation. In contrary, conventional active generation is constrained by a minimum active power generation leading to a bimodal probability distribution, which can be classified into no generation and generation between minimum and maximum power.

\section{B. Voltage Magnitude Uncertainty}

Next, the CPF is applied to determine probability distributions for voltage magnitudes based on the active power dispatch for all MCSs before and after optimization with uncertainty constraints. Diverse probability distributions are identified for the case study (Fig. 9). Some probability distributions can be described by standard normal distributions (Fig. 9a) or skewed normal distributions (Fig. 9b). Others are bimodal (Fig. 9c), which is caused by the uncertain dispatch of conventional power plants. Some extreme cases have bimodal probability distributions spanning across more than $10 \%$ of the rated voltage (Fig. 9d).

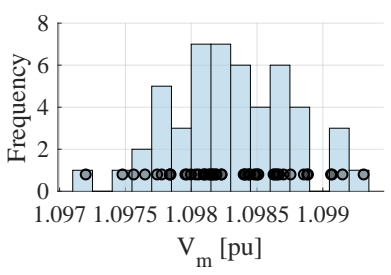

(a) Normal distribution.

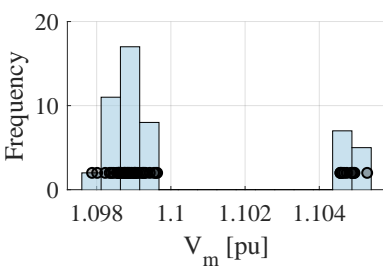

(c) Bimodal distribution (1)

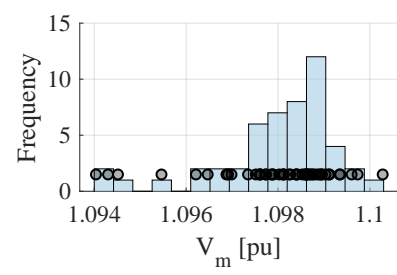

(b) Left skewed distribution.

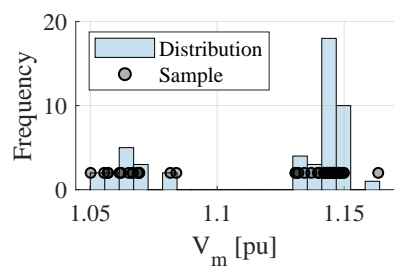

(d) Bimodal distribution (2).
Fig. 9: Exemplary voltage distributions.

The distribution $V_{M}$ for all MCSs and GUCs is visualized in Fig. 10 in reference to $V_{u}$. It shows that the application of uncertainty constraints reduces the probability for voltage limit violations from $3.22 \%$ to $1.08 \%$. In this case study, no violations of the lower voltage limit $V_{l}$ are observed.

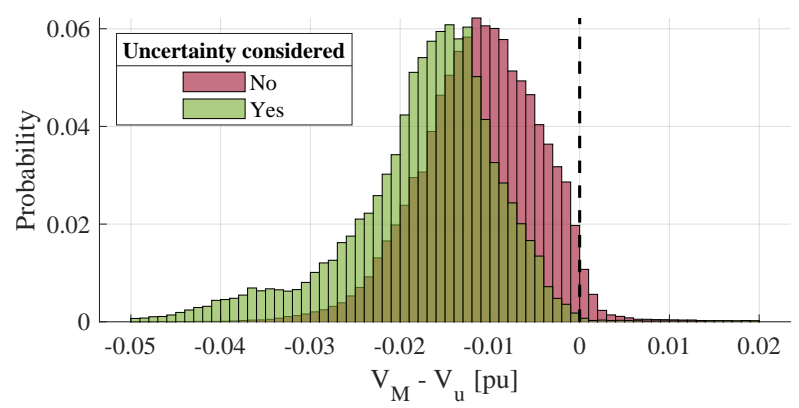

Fig. 10: Difference between $V_{M}$ and the upper limit $V_{u}$.

Exemplary initial voltage magnitude distributions for all buses with voltage limit violations at $t_{15}$ are visualized in Fig. 11.

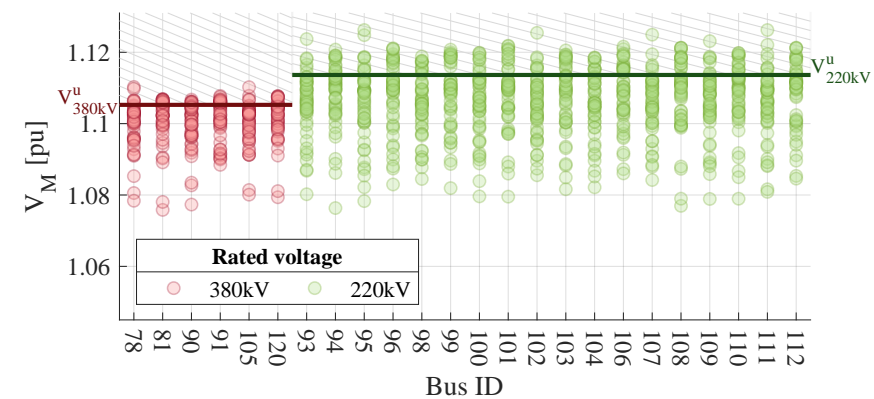

Fig. 11: $V_{M}$ distributions at buses with initial limit violations at $t_{15}$.

Limit violations occur mainly at buses close to uncertain renewable or conventional generation. Active uncertainty constraints shift the voltage magnitude probability distributions and mitigate the risk of voltage limit violations as shown in Fig. 12.

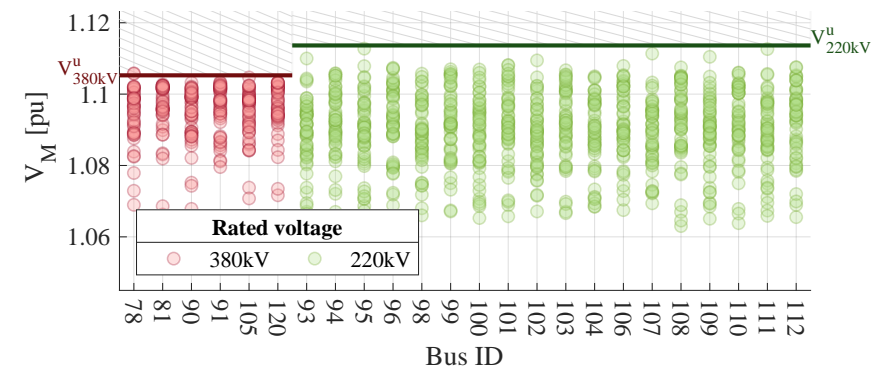

Fig. 12: $V_{M}$ distributions at buses with initial limit violations with active uncertainty constraints at $t_{15}$.

Some MCSs, however, still lead to limit violations. This is a result of using soft constraints, which do not strictly enforce the limits. In this cases, other costs terms (e.g. $c^{L}$ ) outweigh the uncertainty cost term $c^{V, u}$. 


\section{Cost Term Sensitivity}

The enforcement of voltage limits for every possible scenario is not necessarily the major goal of voltage control in transmission system operation. Isolation equipment typically allows temporary overvoltages giving the TSO enough time to react in case of minor violations of $V^{u}$ [17]. The variation of $c^{V, u}$ adapts the method to decrease or increase the priority to mitigate the risk of voltage limit violations. Fig 13 shows the resulting probability for limit violations for different values $c^{V, u}$. Decreasing or increasing $c^{V, u}$ leads to a higher or lower risk of limit violations, respectively. This proves the effectiveness of the implemented approach. However, even at the highest cost terms, a minor chance for voltage limit violations remains. This is caused by the dependency of $\mathcal{P}\left(V_{M}\right)$ on the operating point.

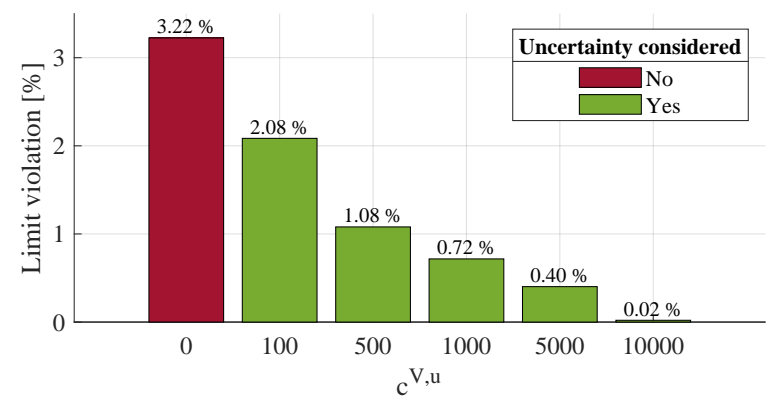

Fig. 13: Sensitivity of cost terms on the probability of voltage limit violations.

\section{DiscusSION AND OUTLOOK}

The developed method shows that skewed or multimodal voltage magnitude probability distributions can be considered for voltage control in transmission grids. However, there is no straightforward approach to model such complex distributions as simple algebraic functions. The modeling as soft constraints enables the prioritization of uncertainty limits in relation to different objectives, such as dispatch costs or active power losses.

So far, the uncertain dispatch of conventional power plants has been neglected. This leads to an over- or underestimation of reactive power reserves. To make the method more robust, a probabilistic modeling of the corresponding variables and variable limits is required. In addition, a probabilistic analysis of long-term voltage stability limits is enabled by the combination of the CPF and Monte Carlo simulations. This will be utilized in future studies, to assess the maximal loading margins for each MCS.

\section{REFERENCES}

[1] E. Lorenz, J. Hurka, G. Karampela, D. Heinemann, H. G. Beyer, and M. Schneider, "Qualified forecast of ensemble power production by spatially dispersed grid-connected pv systems," Measurement, 2007.

[2] Y. Dvorkin, M. Lubin, S. Backhaus, and M. Chertkov, "Uncertainty sets for wind power generation," IEEE Transactions on Power Systems, vol. 31 , no. 4, pp. 3326-3327, 2015.
[3] European Network of Transmission System Operators for Electricity (ENTSO-E). Entso-e transparency platform. [Online]. Available: https://transparency.entsoe.eu/

[4] F. Capitanescu and L. Wehenkel, "Redispatching active and reactive powers using a limited number of control actions," in IEEE Transactions on Power Systems, vol. 26, no. 3. IEEE, 2011, pp. 1221-1230.

[5] M. Lubin, Y. Dvorkin, and S. Backhaus, "A robust approach to chance constrained optimal power flow with renewable generation," IEEE Transactions on Power Systems, vol. 31, no. 5, pp. 3840-3849, 2015.

[6] L. Roald and G. Andersson, "Chance-constrained ac optimal power flow: Reformulations and efficient algorithms," IEEE Transactions on Power Systems, vol. 33, no. 3, pp. 2906-2918, 2017.

[7] S. Granville, "Optimal reactive dispatch through interior point methods," IEEE Transactions on power systems, vol. 9, no. 1, pp. 136-146, 1994.

[8] C. Zhang, H. Chen, Z. Liang, M. Guo, D. Hua, and H. Ngan, "Reactive power optimization under interval uncertainty by the linear approximation method and its modified method," IEEE Transactions on Smart Grid, vol. 9, no. 5, pp. 4587-4600, 2017.

[9] T. Ding, S. Liu, W. Yuan, Z. Bie, and B. Zeng, "A two-stage robust reactive power optimization considering uncertain wind power integration in active distribution networks," IEEE Transactions on Sustainable Energy, vol. 7, no. 1, pp. 301-311, 2015.

[10] N. Qin, C. L. Bak, H. Abildgaard, and Z. Chen, "Multi-stage optimization-based automatic voltage control systems considering wind power forecasting errors," IEEE Transactions on Power Systems, vol. 32, no. 2, pp. 1073-1088, 2016.

[11] M. Knittel, J. Massmann, A. Schnettler, and D. Kamenschikow, "Future operational concepts for reactive power compensators in transmission grids," in Mediterranean Conference on Power Generation, Transmission, Distribution and Energy Conversion (MedPower 2018), 2018.

[12] M. Nobis, J. Clever, N. Thie, and A. Schnettler, "Modeling forecasting errors of fluctuating renewables and electrical loads," in 16th international conference on the European energy market, 092019.

[13] M. Carrión and J. M. Arroyo, "A computationally efficient mixed-integer linear formulation for the thermal unit commitment problem," IEEE Transactions on power systems, vol. 21, no. 3, pp. 1371-1378, 2006.

[14] V. Ajjarapu and C. Christy, "The continuation power flow: a tool for steady state voltage stability analysis," IEEE transactions on Power Systems, vol. 7, no. 1, pp. 416-423, 1992.

[15] R. D. Zimmerman, C. E. Murillo-Sánchez, and R. J. Thomas, "Matpower: Steady-state operations, planning, and analysis tools for power systems research and education," IEEE Transactions on power systems, vol. 26, no. 1, pp. 12-19, 2011.

[16] H. Barrios, A. Roehder, H. Natemeyer, and A. Schnettler, "A benchmark case for network expansion methods," in 2015 IEEE Eindhoven PowerTech. IEEE, 2015.

[17] International Electrotechnical Commission, "IEC 60071-1 Insulation coordination-Part 1: Definitions, principles and rules," IEC Central Office, Geneva, Switzerland, 2010. 